\title{
Linear Prediction Modeling for Evaluating Abnormal Intra QRS Potentials in the High-Resolution Electrocardiogram
}

\author{
CC Lin \\ Department of Electronic Engineering, Lunghwa University of Science and Technology, Taoyuan, \\ Taiwan
}

\begin{abstract}
Background: The abnormal intra-QRS potentials (AIQP) from a signal-averaged electrocardiogram (SAECG) have been proposed to indicate the risk of ventricular arrhythmias. However, the major limitation of current autoregressive moving average modeling is that the model order depends on the database. This study presented a new method based on the linear prediction modeling to improve the limits in AIQP analysis.

Materials and Methods: A total of 154 normal Taiwanese (N), 94 ventricular premature contraction (VPC) patients and 26 sustained ventricular tachycardia (VT) patients were recruited. The AIQP were extracted from the modeling residual of a linear prediction model. From the analyses of all modeling residual curves (modeling residual versus model order), the optimal model order is six. The AIQP was quantified by the rootmean-square value of the modeling residual within $Q R S$ interval.

Results: The AIQP of VT patients were significantly greater than those of non-VT groups (normal and VPC groups) ( $p<0.05)$. No significant differences appeared between normal and VPC groups. A linear combination of AIQP in leads $X, Y$ and $Z$ and three standardized timedomain SAECG parameters provide the best diagnostic performance (specificity $85.9 \%$, sensitivity $88.5 \%$ and predictive accuracy $86.2 \%$ ).

Conclusions: The AIQP can be extracted by the linear prediction modeling to evaluate the risk of ventricular arrhythmias, which can enhance the diagnostic performance of time-domain SAECG. And, the linear prediction modeling improves the clinical feasibility of AIQP analysis.
\end{abstract}

\section{Introduction}

Signal-averaged electrocardiogram (SAECG) has been an important noninvasive technique for evaluating the risk of ventricular arrhythmias [1,2]. An accurate identification of patients with high-risk ventricular arrhythmias is essential for reducing the threat of sudden cardiac death. SAECG has excellent negative predictive accuracy for stratifying the risk of ventricular arrhythmias in patients who are recovering from myocardial infarction, and for identifying patients with ischemic heart disease and unexplained syncope, who are likely to have inducible sustained ventricular tachycardia. However the positive predictive accuracy is not yet sufficiently high to justify interventions for those whose results of analysis are abnormal [3].

SAECG analyses focus on the low-amplitude and highfrequency ventricular late potentials (VLPs) at the terminal QRS complex. VLPs have been approved to be related with the development of sustained ventricular tachycardia (VT) [2,3]. Because VLPs were overlapped with the end of large-amplitude QRS complex, an extremely poor signal-noise-ratio or noise interference can limit VLPs detection.

Apart from the VLPs at the terminal QRS complex, Gomis et al. [4] and Lander et al. [5] presented a new concept - that the abnormal intra-QRS potentials (AIQP) that originate inside the QRS complex may also be an important index of the risk of ventricular arrhythmias. AIQP represent the low-amplitude notches and slurs. An autoregressive moving average (ARMA) model in the discrete cosine transform (DCT) domain was developed to analyze the unpredictable AIQP. It was demonstrated that AIQP can improve the diagnostic performance of SAECG. However, the principal limitation of their methodology is that the order of the model was selected in a data-dependent manner. This limitation reduced the clinical feasibility of AIQP analysis.

This purpose of this study is to develop a linear prediction (LP) modeling in order to improve the limitation of current ARMA modeling in AIQP analysis. The optimal model order needn't be compromised with the diagnostic performance. This study will demonstrate that the AIQP extracted by the LP modeling can effectively evaluate the risk of ventricular arrhythmias and enhance the diagnostic performance of SAECG.

\section{Methods}

There were 154 normal Taiwanese (N), 94 ventricular premature contraction (VPC) patients and 26 sustained 
ventricular tachycardia (VT) patients were recruited in this study. High-resolution ECGs were recorded using a commercially available Simens-Elema Megacart ${ }^{\circledR}$ machine with a bipolar, orthogonal $\mathrm{X}, \mathrm{Y}$ and $\mathrm{Z}$, lead system.

\subsection{Time-domain SAECG analysis}

Three time-domain parameters were calculated - the filtered total QRS duration (fQRSD), the root-meansquare voltage of the last $40 \mathrm{~ms}$ of the QRS complex (RMS40) and the duration of the low amplitude signals below $40 \mu \mathrm{V}$ of the terminal QRS complex (LAS40). The criteria of time-domain SAECG were fQRSD > $114 \mathrm{ms,}$ LAS40 $>38 \mathrm{~ms}$ and RMS40 $<20 \mu \mathrm{V}$ using a 40 to 250 $\mathrm{Hz}$ filter [3]. If any two or more criteria were met, then the VLPs are regarded as present.

\subsection{Linear prediction modeling}

The basic idea of LP modeling of SAECG is that a sampled SAECG data can be approximated as a linear combination of past SAECG samples. The signals $x(n)$ within the entire QRS complex are assumed to contain two main parts - (1) the normal and predictable QRS, $s(n)$, and (2) the unpredictable AIQP, $v(n)$. They are also assumed to be independent of each other. Figure 1 is the block diagram of LP modeling for the analysis of AIQP.

The normal QRS can be estimated by the modeling output which is determined by a set of model coefficients. That is,

$$
\hat{s}(n)=\sum_{i=1}^{m} a(i) x(n-i)
$$

where $a(i)$ is the ith LP model coefficient. These coefficients are used as weighting factors in a linear combination, and $\mathrm{m}$ is the model order.

The difference between the original QRS and the modeling output is the modeling residual $r(n)$ defined as follow.

$$
\begin{aligned}
r(n) & =x(n)-\hat{s}(n) \\
& =x(n)-\sum_{i=1}^{m} a(i) x(n-i)
\end{aligned}
$$

Given a specific model order, the autocorrelation method was adopted to estimate the optimal model coefficients by minimizing the sum of the squares of the modeling residual [6].

Furthermore, the modeling residual can be represented as follows.

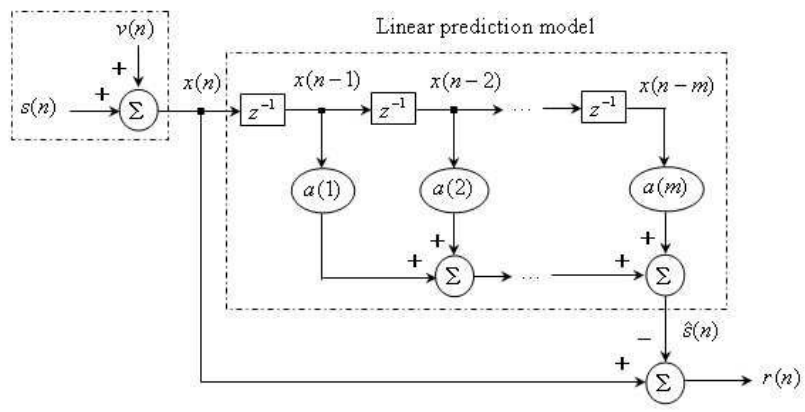

Figure 1. Block diagram of the linear prediction model for analyzing the abnormal intra-QRS potentials.

$$
\begin{aligned}
r(n) & =[v(n)+s(n)]-\hat{s}(n) \\
& =v(n)+e(n)
\end{aligned}
$$

where $e(n)$ is the modeling error that originates from the estimate of the normal QRS. Accordingly, the modeling residual consists of two parts - the AIQP and the modeling error. When the modeling error was reduced as much as possible, the modeling residual can be used to evaluate the unpredictable AIQP.

\subsection{Model order selection}

For application to real SAECG, no a prior knowledge of AIQP characteristics can be used to determine the optimal model order. A model of an excessively high order can still be used to estimate accurately the QRS and AIQP, but also increased the complexity of the calculation of the optimal model coefficients. However, a model of excessively low order can lead to the mixing of the modeling residual into the QRS, influencing the analysis of the AIQP. Accordingly, an appropriate model order is essential for AIQP analysis. In this study, the optimal model order determination was depended on the analyses of the modeling residual curves (modeling residual versus model order). The root-mean-square value of the modeling residual within the QRS interval was used to quantify the modeling residual as follows.

$$
\operatorname{RES}(\mu \mathrm{V})=\sqrt{\frac{1}{Q R S D} \sum_{n=n_{1}}^{n_{2}} r^{2}(n)}
$$

where $n_{1}$ and $n_{2}$ are the onset and offset of the entire QRS complex, respectively. Under the optimal model order and coefficients, the smallest RES was used to quantify the AIQP in leads $\mathrm{X}, \mathrm{Y}$ and $\mathrm{Z}$ (AIQP_l represents AIQP in lead $l, l=\mathrm{X}, \mathrm{Y}$ or $\mathrm{Z}$ ). 


\subsection{Statistical analysis}

Data were presented as mean \pm standard deviation (SD). All statistical analyses were done with Statistical Package for the Social Sciences ${ }^{\circledR}$ (SPSS). Normal distribution tests were performed on all quantitative variables [7]. Statistical significance was defined as $p$ values less than 0.05. Comparisons between pairwise groups were performed using a Student $t$ test for normally distributed continuous variables. Levene's test was used to check the homogeneity of variance between variables. The Mann Whitney $U$ and Wilcoxon Rank Sum tests were used for those non-normally distributed [8].

The definitions of clinical performance indices including specificity (Sp), sensitivity (Se), and total predictive accuracy (TPA) were used [9]. The optimal operation point for the classification of VT and normal subject was determined by the Fisher linear discriminant analysis (LDA) using SPSS for IQP and time-domain parameters.

\section{Results}

\subsection{Modeling residual curves and model order selection}

The analyses of modeling residual curves (RES vs. model order) were performed on all SAECG. Figure 2 plotted the modeling curves of lead Y SAECG for a normal subject (curve 1) and a VT patient (curve 2). These two curves showed that RES did not significantly reduce with the order of the model when the order was higher than 2 and 6 respectively. All of the modeling residual curves showed that the lowest model orders were between 2 and 6 . Hence, selecting a model order beyond six didn't significantly reduce the modeling residual. This study selected a six-order LP model for analyzing the AIQP of all SAECG. The RES of the VT patient was greater than that of the normal subject $(5.6 \mu \mathrm{V}$ vs. $3.2 \mu \mathrm{V})$.

\subsection{Results of time-domain and AIQP analyses}

Table 1 and Table 2 show results of time-domain and AIQP analyses of SAECG. The mean AIQP from leads Y and $\mathrm{Z}$ of the VT group significantly exceeded that of the normal and VPC groups $(\mathrm{p}<0.001)$. The mean AIQP from leads $\mathrm{X}$ of the VT group significantly exceeded that of the normal group, but no significant differences between VT and VPC groups.

The mean fQRSD of the VT group significantly exceeded that of the normal group, but it did not significantly differ from that of the VPC group. The mean LAS40 of VT group significantly exceeded that of both normal and VPC groups. However, the differences of

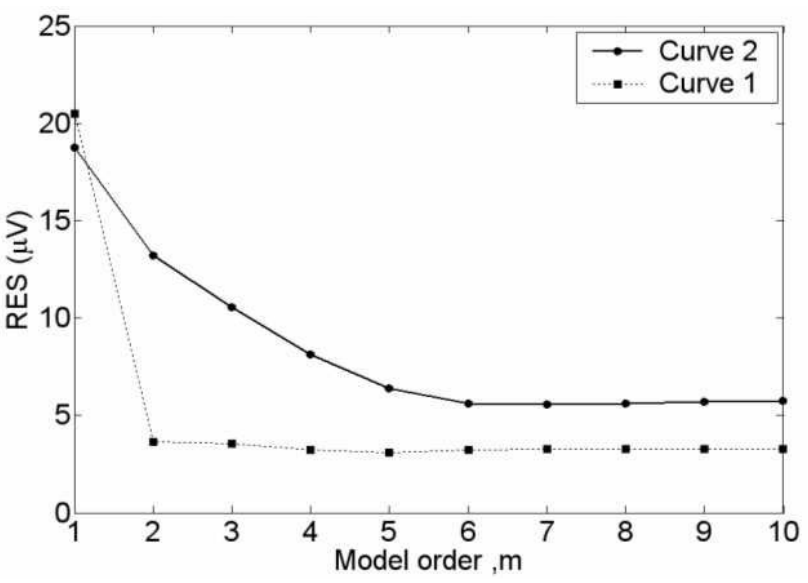

Figure 2. Modeling residual curves of lead Y for a normal subject (curve 1) and a VT patient (curve 2). RES is the root-mean-square value of the modeling residual within QRS duration.

Table 1: Summary of time-domain analysis

\begin{tabular}{cccc}
\hline & \multicolumn{3}{c}{ Time-domain parameters } \\
\cline { 2 - 4 } Subjects & $\begin{array}{c}\text { fQRSD } \\
(\mathrm{ms})\end{array}$ & $\begin{array}{c}\text { LAS40 } \\
(\mathrm{ms})\end{array}$ & $\begin{array}{c}\text { RMS40 } \\
(\mu \mathrm{V})\end{array}$ \\
\hline VT & $95.7 \pm 7.8$ & $37.0 \pm 7.0$ & $20.6 \pm 9.1$ \\
VPC & $92.1 \pm 9.2^{\mathrm{NS}}$ & $31.1 \pm 7.9^{* *}$ & $35.0 \pm 19.8^{* *}$ \\
Normal & $90.4 \pm 8.6^{*}$ & $30.5 \pm 7.4^{* *}$ & $42.2 \pm 25.5^{* *}$ \\
\hline
\end{tabular}

Table 2: Summary of AIQP analysis

\begin{tabular}{cccc}
\hline & \multicolumn{3}{c}{ AIQP parameters $(\mu \mathrm{V})$} \\
\cline { 2 - 4 } Subjects & AIQP_X & AIQP_Y & AIQP_Z \\
\hline VT & $2.9 \pm 1.0$ & $4.8 \pm 0.8$ & $4.3 \pm 1.1$ \\
VPC & $2.6 \pm 0.6^{\mathrm{NS}}$ & $3.8 \pm 0.7^{* *}$ & $3.3 \pm 0.8^{* *}$ \\
Normal & $2.3 \pm 0.6^{*}$ & $3.6 \pm 0.7^{* *}$ & $3.2 \pm 1.0^{* *}$ \\
\hline
\end{tabular}

The equivalent non-parametric Mann Whitney $U$ and Wilcoxon Ranked Sum tests were performed to compare the means between VT and VPC or Normal groups. Abbreviations: NS, not significant ( $\mathrm{p}>0.05)$; ${ }^{*}, \mathrm{p}<0.01$; **, $\mathrm{p}<0.001$; AIQP_1, abnormal intra-QRS potentials from lead 1 , where $1=\mathrm{X}, \mathrm{Y}$ or $\mathrm{Z}$ ). 
fQRSD and LAS40 between the normal and VPC groups were not significant. The mean RMS40 differed significantly among the three study groups $(21.9 \mu \mathrm{V}$ in the VT group $<35.0 \mu \mathrm{V}$ in the VPC group $<44.0 \mu \mathrm{V}$ in the normal group).

A linear combination of the AIQP and time-domain parameters has the best diagnostic performance ( $\mathrm{Se}=$ $88.5 \%, \quad \mathrm{Sp}=85.9 \%$ and $\mathrm{TPA}=86.2 \%$ ) among all individual parameters. The linearly combined parameter using LDA was $-0.177 \times$ AIQP_X $+1.033 \times$ AIQP_Y + $0.432 \times$ AIQP_Z $-0.003 \times$ fQRSD $+0.044 \times$ LAS40 $0.017 \times$ RMS40 -5.362 . The diagnostic criterion was $>$ 0.88 .

\section{Discussion and conclusions}

This study proposed a new method based on the LP modeling to analyze the AIQP. The modeling output and residual were used to estimate the normal predictable QRS and the unpredictable AIQP respectively. Equation 3 showed that the unpredictable AIQP are mixed with the modeling error in the modeling residual. Hence, the modeling error or residual must be minimized in order to reach an accurate estimation of AIQP. The analyses of the modeling residual curves suggested that a six-order LP model is sufficient to obtain the lowest modeling residual for all SAECG modeling. A higher order was not helpful for reducing the modeling residual, but increased the complexity of calculating the optimal model coefficients.

This work demonstrated that the unpredictable AIQP extracted by the LP modeling are useful for the evaluation of the risk of ventricular arrhythmias and improving the diagnostic performance of the SAECG. The AIQP can effectively separate VT and non-VT groups (VPC and normal groups). A linear combination of AIQP and timedomain parameters effectively increased the diagnostic performance of SAECG.

\section{Acknowledgements}

The author would like to thank the staff of the Hemodialysis Unit and patients of Cardiology
Department at Jen-Chi General Hospital for their kind assistance and cooperation for this study. The clinical suggestions and comments from Dr. Ten-Fang Yang (Taipei Medical University, Taipei, Taiwan) are also appreciated.

\section{References}

[1] Simson MB. Use of signals in the terminal QRS complex to identify patients with ventricular tachycardia after myocardial infarction. Circulation 1981; 64: 235-42.

[2] Breithardt G, Cain ME, El-Sherif N, Flowers N, Hombach V, Janse M, et al. Standards for analysis of ventricular late potentials using high-resolution or signal-averaged electrocardiography: a statement by a task force committee of the European Society of Cardiology, the American Heart Association, and the American College of Cardiology. J Am Coll Cardiol 1991; 17: 999-1006.

[3] Cain ME, Anderson JL, Arnsdorf MF, Mason JW, Scheinman MM, Waldo AL. Signal-averaged electrocardiography. J Am Coll Cardiol 1996; 27: 238-49.

[4] Gomis P, Jones DL, Caminal P, Berbari EJ, Lander P. Analysis of abnormal signals within the QRS complex of the high-resolution electrocardiogram. IEEE Trans Biomed Eng 1997; 44: 681-93.

[5] Lander P, Gomis P, Goyal R, Berbari EJ, Caminal P, Lazzara R, et al. Analysis of abnormal intra-QRS potentials. Improved predictive value for arrhythmic events with the signal-averaged electrocardiogram. Circulation 1997; 95: 1386-93.

[6] Hayes MH. Signal modeling, Statistical digital signal processing and modeling. John Wiley \& Sons, Inc, 1996: $129-214$

[7] Chan YH. Biostatistics 101: data presentation. Singapore Med J 2003; 44: 280-5.

[8] Chan YH. Biostatistics 102: quantitative data--parametric \& non-parametric tests. Singapore Med J 2003; 44: 391-6.

Address for correspondence

Chun-Cheng Lin

Department of Electronic Engineering, Lunghwa University of Science and Technology

No.300, Sec. 1, Wanshou Rd., Guishan Shiang, Taoyuan County 333, Taiwan

cclin@mail.lhu.edu.tw 